\title{
Step-like Contrast Structure of Singularly Perturbed Optimal Control Problem
}

\author{
Limeng $\mathbf{W} \mathbf{u}^{1,2 *}$ Mingkang $\mathbf{N i}^{1,2}$ and Haibo $\mathbf{L u}^{1,2}$ \\ ${ }^{1}$ Department of Mathematics, East China Normal University \\ Shanghai 200241, P.R. China \\ ${ }^{2}$ Division of Computational Science, E-Institute of Shanghai Universities, \\ at SJTU, Shanghai 200240, P.R. China
}

\begin{abstract}
In this paper, the existence of step-like contrast structure for a class of singularly perturbed optimal control problem is shown by the contrast structure theory. By means of direct scheme of boundary function method, we construct the uniformly valid asymptotic solution for the singularly perturbed optimal control problem. Finally, an example is presented to show the result.
\end{abstract}

2000 Mathematics Subject Classification. 34B15; 34E15.

Key words and phrases. Singular perturbation; optimal control; contrast structure.

\section{Introduction}

The contrast structure in a singularly perturbed problem is mainly classified as a step-like contrast structure or a spike-like contrast structure (see [1]-[3]). This issue is called as internal layer solution problem in western [4]. A step-like contrast structure problem is only concerned in this paper. Its fundamental characteristics is that there exists an $t^{*}$ (or multiple $\left.t^{*}\right)$ within the domain of interest, which is called as an internal transition time. The position of $t^{*}$ is unknown in advance if there exists an internal layer solution and it needs to be determined thereafter. In the neighborhood of $t^{*}$, the solution $y(t, \mu)$ will have an abrupt structure change and in the different sides of $t^{*}, y(t, \mu)$ will approach to different reduced solutions when the small parameter $\mu \rightarrow 0$. The contrast structure has a strong

\footnotetext{
*Corresponding author. E-mail: neamou123@163.com.
} 
application background. For example, in the study of physics, there are cases that their solutions vary rapidly in the interior of domain.

In recent years, the study on contrast structures is still a hot but difficult research topic in the theory of singularly perturbed problem. In Russia, the most study on contrast structures is by the boundary function method, and in Western, the study on this issue is by the method of dynamic systems or geometric method [5]. More and more scholars begin to pay attention to the contrast structure of variational problem. In [6], [7], the authors consider the contrast structures for the simplest vector variational problem and scalar variational problem. One of the basic difficulties for such a problem is unknown of where an internal transition layer is in advance.

Currently, there are mainly two ways to solve this problem. The first way is through the boundary function method [8]. Usually, this method is applied to necessary or sufficient optimality conditions. The second alternative is through direct scheme of boundary function method, which consists in a direct expansion of the optimal control problem. we will apply the direct scheme to the singularly perturbed optimal control problem. As a result of the scheme, we get a minimizing control sequence, each new control approximation decreases the performance index of the given problem. It should be noted that the direct scheme not only make it easy to obtain the relations for the high-order approximations, but also show the nature of the optimal control problem.

The theory of boundary value problems with integral boundary conditions arises in different areas of applied mathematics and physics. For example, thermal conduction [9], semiconductor problems [10], biomedical science [11], and the references therein. In [12], the authors consider the existence of contrast structure for the following singularly perturbed differential equations with integral boundary conditions

$$
\begin{gathered}
\mu^{2} \frac{d^{2} y}{d t^{2}}=f(t, y), \quad 0<t<1, \\
y(0, \mu)=\int_{0}^{1} h_{1}(y(s, \mu)) d s, \quad y(1, \mu)=\int_{0}^{1} h_{2}(y(s, \mu)) d s,
\end{gathered}
$$

by using of the theory of differential equalities.

In this present paper, the singularly perturbed optimal control problem with integral boundary conditions is considered, we not only prove the existence of step-like contrast structure for the singularly perturbed optimal control problem, but also construct asymptotic solution to the optimal controller and optimal trajectory. 


\section{Problem Formulation}

Consider the singularly perturbed optimal control problem

$$
\left\{\begin{array}{l}
J[u]=\int_{0}^{T} f(y, u, t) d t \rightarrow \min _{u}, \\
\mu \frac{d y}{d t}=u \\
y(0, \mu)=\int_{0}^{T} h_{1}(y(s, \mu)) d s, \quad y(T, \mu)=\int_{0}^{T} h_{2}(y(s, \mu)) d s
\end{array}\right.
$$

where $\mu>0$ is a small parameter.

The following assumptions are fundamental in the theory for the problem in question.

$\left[A_{1}\right]$ Suppose that $f(y, u, t)$ and $h_{i}$ are sufficiently smooth on the domain $D=\{(y, u, t)||$ $y \mid<A, u \in \mathbb{R}, 0 \leq t \leq T\}$, where $A$ is positive constant, $i=1,2$.

$\left[A_{2}\right]$ Suppose that $f_{u^{2}}(y, u, t)>0$ on the domain $D$.

Formally setting $\mu=0$ in (2.1), we obtain the reduced problem

$$
J[\bar{u}]=\int_{0}^{T} f(\bar{y}, \bar{u}, t) \mathrm{d} t \rightarrow \min _{\bar{u}}, \quad \bar{u}=0 .
$$

For our convenience, problem (2.2) can be written in the following equivalent form

$$
J[\bar{u}]=\int_{0}^{T} F(\bar{y}, t) \mathrm{d} t \rightarrow \min _{\bar{y}},
$$

where $F(\bar{y}, t)=f(\bar{y}, 0, t)$.

$\left[A_{3}\right]$ Suppose that there exist two isolated functions $\bar{y}=\varphi_{1}(t), \bar{y}=\varphi_{2}(t)$ such that

$$
\begin{gathered}
\min _{\bar{y}} F(\bar{y}, t)=\left\{\begin{array}{l}
F\left(\varphi_{1}(t), t\right) \quad 0 \leq t \leq t_{0}, \\
F\left(\varphi_{2}(t), t\right), \quad t_{0} \leq t \leq T,
\end{array}\right. \\
\lim _{t \rightarrow t_{0}^{-}} \varphi_{1}(t) \neq \lim _{t \rightarrow t_{0}^{+}} \varphi_{2}(t) .
\end{gathered}
$$

$\left[A_{4}\right]$ Suppose that the transition point $t_{0}$ is determined by the following equation

$$
F\left(\varphi_{1}\left(t_{0}\right), t_{0}\right)=F\left(\varphi_{2}\left(t_{0}\right), t_{0}\right),
$$

and satisfies the condition

$$
\frac{d}{d t} F\left(\varphi_{1}\left(t_{0}\right), t_{0}\right) \neq \frac{d}{d t} F\left(\varphi_{2}\left(t_{0}\right), t_{0}\right) .
$$

EJQTDE, 2011 No. 46, p. 3 
It follows from assumption $\left[A_{3}\right]$ that

$$
\left\{\begin{array}{lll}
F_{y}\left(\varphi_{1}(t), t\right)=0, & F_{y y}\left(\varphi_{1}(t), t\right)>0, & 0 \leq t \leq t_{0} \\
F_{y}\left(\varphi_{2}(t), t\right)=0, & F_{y y}\left(\varphi_{2}(t), t\right)>0, & t_{0} \leq t \leq T .
\end{array}\right.
$$

Consider the Hamiltonian function

$$
H(y, u, \lambda, t)=f(y, u, t)+\lambda \mu^{-1} u,
$$

where $\lambda$ is Lagrange multiplier.

The necessary optimality conditions imply that

$$
\left\{\begin{array}{l}
\mu y^{\prime}=u, \\
\lambda^{\prime}=-f_{y}(y, u, t), \\
\mu f_{u}(y, u, t)+\lambda(t)=0, \\
y(0, \mu)=\int_{0}^{T} h_{1}(y(s, \mu)) d s, \quad y(T, \mu)=\int_{0}^{T} h_{2}(y(s, \mu)) d s .
\end{array}\right.
$$

From (2.5), we can obtain the following singularly perturbed boundary value problem

$$
\left\{\begin{array}{l}
\mu y^{\prime}=u \\
\mu u^{\prime}=f_{u^{2}}^{-1}\left(f_{y}-f_{u y} u\right)-\mu f_{u^{2}}^{-1} f_{u t} \\
y(0, \mu)=\int_{0}^{T} h_{1}(y(s, \mu)) d s, \quad y(T, \mu)=\int_{0}^{T} h_{2}(y(s, \mu)) d s
\end{array}\right.
$$

problem of type (2.6) was considered in [12], in which the existence of solution with steplike contrast structure was shown. By means of the result as described in [12], we show the existence of optimal trajectory with step-like contrast structure.

\section{Existence of Step-like Contrast Structure}

As was mentioned above, problem of type (2.6) was considered in [12], therefore, under suitable conditions, the extremal trajectory (the solution to the system of Euler equations (2.6)) contains a step-like contrast structure.

It is easy to see that the associated system for (2.6) can be written as

$$
\left\{\begin{array}{l}
\frac{d u}{d \tau}=f_{u^{2}}^{-1}\left(f_{y}-f_{u y} u\right), \\
\frac{d y}{d \tau}=u
\end{array}\right.
$$

Now we will state and prove some useful lemmas, which we will use to prove our main results. We begin with the following lemma. 
Lemma 3.1 Suppose that $\left[A_{1}\right]-\left[A_{4}\right]$ hold. Then associated system (3.1) has two equilibria $M_{i}\left(\varphi_{i}(\bar{t}), 0\right), i=1,2$, which are both saddle points.

Proof. Let

$$
\begin{gathered}
H(y, u, \bar{t})=f_{u^{2}}^{-1}\left(f_{y}-f_{u y} u\right), \\
G(y, u, \bar{t})=u .
\end{gathered}
$$

Obviously, $M_{i}\left(\varphi_{i}(\bar{t}), 0\right), i=1,2$ are two isolated solutions of the reduced system

$$
\begin{aligned}
H(y, u, \bar{t}) & =0, \\
G(y, u, \bar{t}) & =0 .
\end{aligned}
$$

Moreover, the characteristic equation of the system (3.1) is given by

$$
\lambda^{2}-\frac{\bar{f}_{y^{2}}}{\bar{f}_{u^{2}}}=0
$$

where $\bar{f}_{y^{2}}$, and $\bar{f}_{u^{2}}$ are calculated in $\left(\varphi_{i}(\bar{t}), 0\right), i=1,2$. Using assumption $(2.4)$, we obtain

$$
\lambda^{2}=\frac{\bar{f}_{y^{2}}}{\bar{f}_{u^{2}}}>0 .
$$

Hence, in the phase plane $(y, u), M_{i}\left(\varphi_{i}(\bar{t}), 0\right), i=1,2$ are both saddle points.

Lemma 3.2 For fixed $\bar{t} \in[0, T]$, associated system (3.1) has a first integral

$$
u f_{u}(y, u, \bar{t})-f(y, u, \bar{t})=C,
$$

where $C$ is a constant.

Proof. Let $y^{\prime}=\frac{d y}{d \tau}, u^{\prime}=\frac{d u}{d \tau}$. Then the first equation in (3.1) can be written as

$$
f_{u^{2}}(y, u, \bar{t}) u^{\prime}=f_{y}(y, u, \bar{t})-f_{u y}(y, u, \bar{t}) u
$$

using the second equation of (3.1), we get

$$
f_{u^{2}}(y, u, \bar{t}) u^{\prime}-f_{y}(y, u, \bar{t})+f_{u y}(y, u, \bar{t}) y^{\prime}=0 .
$$

In view of $y^{\prime \prime}=u^{\prime}$, we obtain

$$
\frac{d}{d \tau}\left(y^{\prime} f_{u}(y, u, \bar{t})-f(y, u, \bar{t})\right)=0 .
$$

Therefore, the first integral for (3.1) is

$$
u f_{u}(y, u, \bar{t})-f(y, u, \bar{t})=C,
$$

where $C$ is a constant. 
Lemma 3.3 Suppose that $\left[A_{1}\right],\left[A_{2}\right]$ and $u \neq 0$ hold. Then, for fixed $\bar{t} \in[0, T]$, the first integral (3.2) is solvable with respect to $u$.

Proof. Let

$$
G(y, u, \bar{t})=u f_{u}(y, u, \bar{t})-f(y, u, \bar{t})-C,
$$

Obviously

$$
G_{u}(y, u, \bar{t})=f_{u}(y, u, \bar{t})+u f_{u^{2}}(y, u, \bar{t})-f_{u}(y, u, \bar{t})=u f_{u^{2}} \neq 0
$$

by the implicit function theorem, the equation $G(y, u, \bar{t})=0$ is solvable with respect to $u$ :

$$
u=h(y, \bar{t}, C), \quad(y, \bar{t}) \in D_{1} .
$$

where $D_{1}=\{(y, t)|| y \mid<A, 0 \leq t \leq T\}$.

let us continue the verification of the assumptions of [12]. Obviously, there exist two separate orbits $S_{M_{1}}$ and $S_{M_{1}}$ that pass through the saddle points $M_{1}$ and $M_{2}$, which satisfy the equations

$$
\begin{aligned}
& S_{M_{1}}: \quad u f_{u}(y, u, \bar{t})-f(y, u, \bar{t})=-f\left(\varphi_{1}(\bar{t}), 0, \bar{t}\right), \\
& S_{M_{2}}: \quad u f_{u}(y, u, \bar{t})-f(y, u, \bar{t})=-f\left(\varphi_{2}(\bar{t}), 0, \bar{t}\right) .
\end{aligned}
$$

It follows from Lemma 3.3 that

$$
\begin{aligned}
& u^{(-)}(\tau, \bar{t})=h^{(-)}\left(y^{(-)}, \bar{t}, \varphi_{1}(\bar{t})\right), \\
& u^{(+)}(\tau, \bar{t})=h^{(+)}\left(y^{(+)}, \bar{t}, \varphi_{2}(\bar{t})\right) .
\end{aligned}
$$

Let

$$
H(\bar{t})=u^{(-)}(0, \bar{t})-u^{(+)}(0, \bar{t})=h^{(-)}\left(y^{(-)}(0), \bar{t}, \varphi_{1}(\bar{t})\right)-h^{(+)}\left(y^{(+)}(0), \bar{t}, \varphi_{2}(\bar{t})\right),
$$

where $y^{(-)}(0)=y^{(+)}(0)=\frac{1}{2}\left(\varphi_{1}(\bar{t})+\varphi_{2}(\bar{t})\right)=\beta(\bar{t})$.

Lemma 3.4 Suppose that $\left[A_{1}\right]-\left[A_{4}\right]$ hold. Then, we get

$$
h_{y}\left(\varphi_{i}(\bar{t}), \bar{t}\right)= \pm \sqrt{\frac{f_{y^{2}}\left(\varphi_{i}(\bar{t}), 0, \bar{t}\right)}{f_{u^{2}}\left(\varphi_{i}(\bar{t}), 0, \bar{t}\right)}}, \quad i=1,2 .
$$


Proof. Differentiating the implicit function, we have

$$
h_{y}(y, \bar{t})=\frac{d u}{d y}=\frac{f_{y}-u f_{u y}}{u f_{u^{2}}} .
$$

Using L'Hospital's rule, in the neighborhood of saddle points, we obtain

$$
h_{y}\left(\varphi_{i}(\bar{t}), \bar{t}\right)= \pm \sqrt{\frac{f_{y^{2}}\left(\varphi_{i}(\bar{t}), 0, \bar{t}\right)}{f_{u^{2}}\left(\varphi_{i}(\bar{t}), 0, \bar{t}\right)}}, \quad i=1,2 .
$$

Lemma 3.5 Suppose that $\left[A_{1}\right]-\left[A_{4}\right]$ hold. Then $H\left(t_{0}\right)=0$ if and only if

$$
f\left(\varphi_{1}\left(t_{0}\right), 0, t_{0}\right)=f\left(\varphi_{2}\left(t_{0}\right), 0, t_{0}\right)
$$

Proof. Setting $\tau=0, \bar{t}=t_{0}$ in (3.6) and (3.7), we obtain

$$
\begin{aligned}
& h^{(-)}\left(t_{0}\right) f_{u}\left(\beta\left(t_{0}\right), h^{(-)}\left(t_{0}\right), t_{0}\right)-f\left(\beta\left(t_{0}\right), h^{(-)}\left(t_{0}\right), t_{0}\right)=-f\left(\varphi_{1}\left(t_{0}\right), 0, t_{0}\right), \\
& h^{(+)}\left(t_{0}\right) f_{u}\left(\beta\left(t_{0}\right), h^{(+)}\left(t_{0}\right), t_{0}\right)-f\left(\beta\left(t_{0}\right), h^{(+)}\left(t_{0}\right), t_{0}\right)=-f\left(\varphi_{2}\left(t_{0}\right), 0, t_{0}\right),
\end{aligned}
$$

where

$$
h^{(-)}\left(t_{0}\right)=h^{(-)}\left(\beta\left(t_{0}\right), \varphi_{1}\left(t_{0}\right), t_{0}\right), \quad h^{(+)}\left(t_{0}\right)=h^{(+)}\left(\beta\left(t_{0}\right), \varphi_{2}\left(t_{0}\right), t_{0}\right),
$$

Necessity follows directly from (3.10), (3.11) and sufficiency follows from (3.5).

Lemma 3.6 Suppose that $\left[A_{1}\right]-\left[A_{4}\right]$ hold. Then $\frac{d}{d t} H\left(t_{0}\right) \neq 0$ if and only if

$$
\frac{d}{d t} f\left(\varphi_{1}\left(t_{0}\right), 0, t_{0}\right) \neq \frac{d}{d t} f\left(\varphi_{2}\left(t_{0}\right), 0, t_{0}\right)
$$

Proof. Setting $\tau=0$ in (3.6) and (3.7), we get

$$
\begin{aligned}
& h^{(-)}(\bar{t}) f_{u}\left(\beta(\bar{t}), h^{(-)}(\bar{t}), \bar{t}\right)-f\left(\beta(\bar{t}), h^{(-)}(\bar{t}), \bar{t}\right)=-f\left(\varphi_{1}(\bar{t}), 0, \bar{t}\right), \\
& h^{(+)}(\bar{t}) f_{u}\left(\beta(\bar{t}), h^{(+)}(\bar{t}), \bar{t}\right)-f\left(\beta(\bar{t}), h^{(+)}(\bar{t}), \bar{t}\right)=-f\left(\varphi_{2}(\bar{t}), 0, \bar{t}\right),
\end{aligned}
$$

where

$$
h^{(-)}(\bar{t})=h^{(-)}\left(\beta(\bar{t}), \varphi_{1}(\bar{t}), \bar{t}\right), \quad h^{(+)}(\bar{t})=h^{(+)}\left(\beta(\bar{t}), \varphi_{2}(\bar{t}), \bar{t}\right),
$$

Differentiating (3.12) and (3.13) with respect to $\bar{t}$, we obtain

$$
\frac{d}{d \bar{t}}\left(h^{(-)}(\bar{t})\right) f_{u}\left(\beta(\bar{t}), h^{(-)}(\bar{t}), \bar{t}\right)+h^{(-)}(\bar{t}) \frac{d}{d \bar{t}}\left(f_{u}\left(\beta(\bar{t}), h^{(-)}(\bar{t}), \bar{t}\right)\right)
$$




$$
\begin{aligned}
&-\frac{d}{d \bar{t}} f\left(\beta(\bar{t}), h^{(-)}(\bar{t}), \bar{t}\right)=-\frac{d}{d \bar{t}} f\left(\varphi_{1}(\bar{t}), 0, \bar{t}\right), \\
& \frac{d}{d \bar{t}}\left(h^{(+)}(\bar{t})\right) f_{u}\left(\beta(\bar{t}), h^{(+)}(\bar{t}), \bar{t}\right)+h^{(+)}(\bar{t}) \frac{d}{d \bar{t}}\left(f_{u}\left(\beta(\bar{t}), h^{(+)}(\bar{t}), \bar{t}\right)\right) \\
&-\frac{d}{d \bar{t}} f\left(\beta(\bar{t}), h^{(+)}(\bar{t}), \bar{t}\right)=-\frac{d}{d \bar{t}} f\left(\varphi_{2}(\bar{t}), 0, \bar{t}\right),
\end{aligned}
$$

let $\bar{t}=t_{0}$ yields

$$
\begin{aligned}
& h^{(-)}\left(t_{0}\right) f_{u^{2}}\left(\beta\left(t_{0}\right), h^{(-)}\left(t_{0}\right), t_{0}\right) \frac{d}{d \bar{t}} H\left(t_{0}\right) \\
= & -\left(\frac{d}{d \bar{t}} f\left(\varphi_{1}\left(t_{0}\right), 0, t_{0}\right)-\frac{d}{d \bar{t}} f\left(\varphi_{2}\left(t_{0}\right), 0, t_{0}\right)\right) .
\end{aligned}
$$

From assumptions $\left[A_{1}\right]$ and $\left[A_{2}\right]$, also since the different orbits do not intersect the line $\bar{u}=0$ at the point $y=\beta\left(t_{0}\right)$, hence $\frac{d}{d t} H\left(t_{0}\right) \neq 0$ if and only if

$$
\frac{d}{d t} f\left(\varphi_{1}\left(t_{0}\right), 0, t_{0}\right) \neq \frac{d}{d t} f\left(\varphi_{2}\left(t_{0}\right), 0, t_{0}\right)
$$

From Lemma 3.2 and Lemma 3.5, it is easy for us to get the next Lemma.

Lemma 3.7 Suppose that $\left[A_{1}\right]-\left[A_{4}\right]$ hold. Then there exists $\bar{t}=t_{0}$ at which associated system (3.1) has a heteroclinic orbit connecting saddle points $M_{1}\left(\varphi_{1}\left(t_{0}\right), 0\right)$ and $M_{2}\left(\varphi_{2}\left(t_{0}\right), 0\right)$.

From the above discussions, we know that the boundary value problem (2.6) satisfies all the assumptions of [12]. Then problem $(2.1)$ has an extremal trajectory $y(t, \mu)$ with a step-like contrast structure. From Theorem 3.3 of [12], we can obtain the following theorem.

Theorem 3.8 Suppose that $\left[A_{1}\right]-\left[A_{4}\right]$ hold. Then for sufficiently small $\mu>0$, the optimal control problem (2.1) has an extremal trajectory $y(t, \mu)$ with a step-like contrast structure

$$
\lim _{\mu \rightarrow 0} y(t, \mu)=\left\{\begin{array}{l}
\varphi_{1}(t), 0 \leq t<t_{0} \\
\varphi_{2}(t), t_{0}<t \leq T .
\end{array}\right.
$$

\section{Construction of Asymptotic Solution}

an asymptotic solution of problem (2.1) is sought in the form

$$
\left\{\begin{array}{l}
y(t, \mu)=\sum_{k=0}^{\infty} \mu^{k}\left(\bar{y}_{k}(t)+L_{k} y\left(\tau_{0}\right)+Q_{0}^{(-)} y(\tau)\right), \quad 0 \leq t<t^{*}, \\
u(t, \mu)=\sum_{k=0}^{\infty} \mu^{k}\left(\bar{u}_{k}(t)+L_{k} u\left(\tau_{0}\right)+Q_{0}^{(-)} u(\tau)\right),
\end{array}\right.
$$

EJQTDE, 2011 No. 46, p. 8 


$$
\left\{\begin{array}{l}
y(t, \mu)=\sum_{k=0}^{\infty} \mu^{k}\left(\bar{y}_{k}(t)+Q_{0}^{(+)} y(\tau)+R_{k} y\left(\tau_{1}\right)\right), \quad t^{*}<t \leq T \\
u(t, \mu)=\sum_{k=0}^{\infty} \mu^{k}\left(\bar{u}_{k}(t)+Q_{0}^{(+)} u(\tau)+R_{k} u\left(\tau_{1}\right)\right)
\end{array}\right.
$$

where $\tau_{0}=t \mu^{-1}, \quad \tau=\left(t-t^{*}\right) \mu^{-1}, \quad \tau_{1}=(t-T) \mu^{-1}, L_{k} y\left(\tau_{0}\right)$ are coefficients of boundary layer terms at $t=0, R_{k}\left(\tau_{1}\right)$ are coefficients of boundary layer terms at $t=T, Q_{k}^{(\mp)}(\tau)$ are left and right coefficients of internal transition terms at $t=t^{*}$.

The position of a transition time $t^{*}(\mu) \in[0, T]$ is unknown in advance. Suppose that $t^{*}$ has also asymptotic expression of the form $t^{*}=t_{0}+\mu t_{1}+\cdots+\mu^{k} t_{k}+\cdots$. The coefficients of the above series are determined during the construction of an asymptotic solution.

From the main results of [6], we obtain

$$
\min _{u} J[u]=\min _{u_{0}} J\left(u_{0}\right)+\sum_{i=1}^{n} \mu^{i} \min _{u_{i}} \tilde{J}_{i}\left(u_{i}\right)+\cdots,
$$

where $\tilde{J}_{i}\left(u_{i}\right)=J_{i}\left(u_{i}, \tilde{u}_{i-1}, \cdots, \tilde{u}_{0}\right), \tilde{u}_{k}=\arg \left(\min _{u_{k}} \tilde{J}_{k}\left(u_{k}\right)\right), k=\overline{0, i-1}$.

Substituting (4.1), (4.2) into (2.1) and equating separately the terms on $t, \tau_{0}, \tau$ and $\tau_{1}$ by the boundary function method, we can obtain a series of variational problems to determine $\left\{\bar{y}_{k}(t), \bar{u}_{k}(t)\right\},\left\{L_{k} y\left(\tau_{0}\right), L_{k} u\left(\tau_{0}\right)\right\},\left\{Q_{k}^{\mp} y(\tau), Q_{k}^{\mp} u(\tau)\right\},\left\{R_{k} y\left(\tau_{1}\right), R_{k} u\left(\tau_{1}\right)\right\}, k \geq 0$ respectively.

The variational problem to determine the zero-order coefficients of regular terms $\left\{\bar{y}_{0}(t)\right.$, $\left.\bar{u}_{0}(t)\right\}$ are given by

$$
\left\{\begin{array}{l}
J_{0}\left(\bar{u}_{0}\right)=\int_{0}^{T} f\left(\bar{y}_{0}, \bar{u}_{0}, t\right) d t \rightarrow \min _{\bar{u}_{0}} \\
\bar{u}_{0}=0
\end{array}\right.
$$

By assumption $\left[A_{3}\right]$, we get

$$
\begin{gathered}
\bar{y}_{0}=\left\{\begin{array}{l}
\varphi_{1}(t), 0 \leq t<t_{0}, \\
\varphi_{2}(t), t_{0}<t \leq T,
\end{array}\right. \\
\bar{u}_{0}=\left\{\begin{array}{l}
0,0 \leq t<t_{0} \\
0, t_{0}<t \leq T
\end{array}\right.
\end{gathered}
$$


The following variational problems to determine $\left\{Q_{0}^{(\mp)} y(\tau), Q_{0}^{(\mp)} u(\tau)\right\}$ are given by

$$
\left\{\begin{array}{l}
Q_{0}^{(\mp)} J=\int_{-\infty(0)}^{0(+\infty)} \Delta_{0}^{(\mp)} f\left(\varphi_{1,2}\left(t_{0}\right)+Q_{0}^{(\mp)} y, \alpha_{1,2}\left(t_{0}\right)+Q_{0}^{(\mp)} u, t_{0}\right) d \tau \rightarrow \min _{Q_{0}^{(\mp)} u}, \\
\frac{d}{d \tau} Q_{0}^{(\mp)} y=Q_{0}^{(\mp)} u, \\
Q_{0}^{(\mp)} y(0)=\beta\left(t_{0}\right)-\varphi_{1,2}\left(t_{0}\right), \quad Q_{0}^{(\mp)} y(\mp \infty)=0,
\end{array}\right.
$$

where

$$
\Delta_{0}^{(\mp)} f=f\left(\varphi_{1,2}\left(t_{0}\right)+Q_{0}^{(\mp)} y, Q_{0}^{(\mp)} u, t_{0}\right)-f\left(\varphi_{1,2}\left(t_{0}\right), 0, t_{0}\right) .
$$

Making the substitutions

$$
\tilde{y}^{(\mp)}=\varphi_{1,2}\left(t_{0}\right)+Q_{0}^{(\mp)} y(\tau), \quad \tilde{u}^{(\mp)}=Q_{0}^{(\mp)} u(\tau) .
$$

we obtain

$$
\left\{\begin{array}{l}
Q_{0}^{(\mp)} J=\int_{-\infty(0)}^{0(+\infty)} \Delta_{0}^{(\mp)} \tilde{f}\left(\tilde{y}^{(\mp)}(\tau), \tilde{u}^{(\mp)}(\tau), t_{0}\right) d \tau \rightarrow \min _{\tilde{u}^{(\mp)}\left(\tilde{y}^{(\mp)}\right)}, \\
\frac{d \tilde{y}^{(\mp)}}{d \tau}=\tilde{u}^{(\mp)}, \\
\tilde{y}^{(\mp)}(0)=\beta\left(t_{0}\right), \quad \tilde{y}^{(\mp)}(\mp \infty)=\varphi_{1,2}\left(t_{0}\right) .
\end{array}\right.
$$

The substitution

$$
\frac{d \tilde{y}^{(\mp)}}{\tilde{u}^{(\mp)}}=d \tau
$$

produces the following variational problem, which is explicitly independent of $\tau$

$$
Q_{0}^{(\mp)} J=\int_{\varphi_{1}\left(t_{0}\right)\left(\beta\left(t_{0}\right)\right)}^{\beta\left(t_{0}\right)\left(\varphi_{2}\left(t_{0}\right)\right)} \frac{\Delta_{0} \tilde{f}\left(\tilde{y}^{(\mp)}, \tilde{u}^{(\mp)}, t_{0}\right)}{\tilde{u}^{(\mp)}} d \tilde{y} \rightarrow \min _{\tilde{u}^{(\mp)}\left(\tilde{y}^{(\mp)}\right)},
$$

the necessary condition for a minimum of the integrand has the form

$$
\tilde{u}^{(\mp)} f_{u}\left(\tilde{y}^{(\mp)}, \tilde{u}^{(\mp)}, t_{0}\right)-f\left(\tilde{y}^{(\mp)}, \tilde{u}^{(\mp)}, t_{0}\right)=-f\left(\varphi_{1,2}\left(t_{0}\right), 0, t_{0}\right),
$$

in view of (3.6) and (3.7), we see that $\tilde{u}^{(\mp)}=h^{(\mp)}\left(\tilde{y}^{(\mp)}, t_{0}\right)$ is the minimum, since it satisfies

$$
\frac{\tilde{u}^{(\mp)} f_{u^{2}}\left(\tilde{y}^{(\mp)}, \tilde{u}^{(\mp)}, t_{0}\right)}{\tilde{u}^{(\mp) 2}}>0 .
$$

From the necessary condition of (4.5), we have

$$
\tilde{u}^{(\mp)} f_{u}\left(\tilde{y}^{(\mp)}, \tilde{u}^{(\mp)}, t_{0}\right)-f\left(\tilde{y}^{(\mp)}, \tilde{u}^{(\mp)}, t_{0}\right)=-f\left(\varphi_{1,2}\left(t_{0}\right), 0, t_{0}\right),
$$

by lemma 3.3, we can obtain $\tilde{u}^{(\mp)}=h^{(\mp)}\left(\tilde{y}, t_{0}\right)$. Using the second expression of (4.4) and the conditions of (4.4), it is easy for us to get the following initial problems

$$
\left\{\begin{array}{l}
\frac{d Q_{0}^{(\mp)} y}{d \tau}=h^{(\mp)}\left(\varphi_{1,2}\left(t_{0}\right)+Q_{0}^{(\mp)} y, t_{0}\right), \\
Q_{0}^{(\mp)} y(0)=\beta\left(t_{0}\right)-\varphi_{1,2}\left(t_{0}\right)
\end{array}\right.
$$

EJQTDE, 2011 No. 46, p. 10 
Here the $Q_{0}^{(\mp)} y(\tau), \quad-\infty<\tau<+\infty$ are determined.

Substituting $Q_{0}^{(\mp)} y(\tau)$ into (4.3), we can obtain $Q_{0}^{(\mp)} u(\tau)$, thus $Q_{0}^{(\mp)} y(\tau)$ and $Q_{0}^{(\mp)} u(\tau)$ are determined. From Lemma 3.4 we get $h_{y}^{(-)}\left(\varphi_{1}\left(t_{0}\right), t_{0}\right)>0, h_{y}^{(+)}\left(\varphi_{2}\left(t_{0}\right), t_{0}\right)<0$, which imply that

$$
\begin{gathered}
\left|Q_{0}^{(-)} y(\tau)\right| \leq C_{0}^{(-)} e^{\kappa_{0} \tau}, \kappa_{0}>0, \tau<0, \\
\left|Q_{0}^{(+)} y(\tau)\right| \leq C_{0}^{(+)} e^{-\kappa_{1} \tau}, \kappa_{1}>0, \tau>0, \\
\left|Q_{0}^{(-)} u(\tau)\right| \leq C_{1}^{(-)} e^{\kappa_{0} \tau}, \kappa_{0}>0, \tau<0, \\
\left|Q_{0}^{(+)} u(\tau)\right| \leq C_{1}^{(+)} e^{-\kappa_{1} \tau}, \kappa_{1}>0, \tau>0 .
\end{gathered}
$$

Next, we give the equations and their conditions for determining $\left\{L_{0} y^{*}\left(\tau_{0}\right), L_{0} u^{*}\left(\tau_{0}\right)\right\}$ as follows

$$
\left\{\begin{array}{l}
L_{0} J=\int_{0}^{\infty} \Delta_{0} f\left(\varphi_{1}(0)+L_{0} y, L_{0} u, 0\right) d \tau_{0} \rightarrow \min _{L_{0} u} \\
\frac{d}{d \tau_{0}} L_{0} y=L_{0} u \\
L_{0} y(0)=\int_{0}^{t_{0}} h_{1}\left(\varphi_{1}(s)\right) \mathrm{d} s+\int_{t_{0}}^{T} h_{1}\left(\varphi_{2}(s)\right) \mathrm{d} s-\varphi_{1}(0), \quad L_{0} y(\infty)=0
\end{array}\right.
$$

where

$$
\Delta_{0} f=f\left(\varphi_{1}(0)+L_{0} y, L_{0} u, 0\right)-f\left(\varphi_{1}(0), 0,0\right),
$$

and the problem to determine $\left\{R_{0} y^{*}\left(\tau_{1}\right), R_{0} u^{*}\left(\tau_{1}\right)\right\}$ is given by

$$
\left\{\begin{array}{l}
R_{0} J=\int_{-\infty}^{0} \Delta_{0} f\left(\varphi_{2}(T)+R_{0} y, R_{0} u, T\right) d \tau_{1} \rightarrow \min _{R_{0} u}, \\
\frac{d}{d \tau_{1}} R_{0} y=R_{0} u \\
R_{0} y(0)=\int_{0}^{t_{0}} h_{2}\left(\varphi_{1}(s)\right) \mathrm{d} s+\int_{t_{0}}^{T} h_{2}\left(\varphi_{2}(s)\right) \mathrm{d} s-\varphi_{2}(T), \quad R_{0} y(-\infty)=0,
\end{array}\right.
$$

where

$$
\Delta_{0} f=f\left(\varphi_{2}(T)+R_{0} y, R_{0} u, T\right)-f\left(\varphi_{2}(T), 0, T\right) .
$$

Similarly as the discussions of $Q_{0}^{(\mp)} y(\tau)$, we can get the following initial problems

$$
\left\{\begin{array}{l}
\frac{d L_{0} y}{d \tau_{0}}=h\left(\varphi_{1}(0)+L_{0} y, 0\right) \\
L_{0} y(0)=\int_{0}^{t_{0}} h_{1}\left(\varphi_{1}(s)\right) \mathrm{d} s+\int_{t_{0}}^{T} h_{1}\left(\varphi_{2}(s)\right) \mathrm{d} s-\varphi_{1}(0)
\end{array}\right.
$$

and

$$
\left\{\begin{array}{l}
\frac{d R_{0} y}{d \tau_{1}}=h\left(\varphi_{2}(T)+L_{0} y, T\right), \\
R_{0} y(0)=\int_{0}^{t_{0}} h_{2}\left(\varphi_{1}(s)\right) \mathrm{d} s+\int_{t_{0}}^{T} h_{2}\left(\varphi_{2}(s)\right) \mathrm{d} s-\varphi_{2}(T),
\end{array}\right.
$$


Here the $L_{0} y\left(\tau_{0}\right)$ and $R_{0} y\left(\tau_{1}\right), 0 \leq \tau_{0}<+\infty,-\infty<\tau_{1} \leq 0$ are determined.

Substituting $L_{0} y\left(\tau_{0}\right)$ into (4.6) and $R_{0} y\left(\tau_{1}\right)$ into (4.7), it is easy for us to get $L_{0} u\left(\tau_{0}\right)$ and $R_{0} u\left(\tau_{1}\right)$, thus $L_{0} y\left(\tau_{0}\right), R_{0} y\left(\tau_{1}\right), L_{0} u\left(\tau_{0}\right)$ and $R_{0} u\left(\tau_{1}\right)$ are determined. From Lemma 3.4 we get $h_{y}\left(\varphi_{1}(0), 0\right)<0, h_{y}\left(\varphi_{2}(T), T\right)>0$, which imply that

$$
\begin{gathered}
\left|L_{0} y\left(\tau_{0}\right)\right| \leq C_{2}^{(-)} e^{-\kappa_{2} \tau_{0}}, \kappa_{2}>0, \tau_{0}>0, \\
\left|R_{0} y\left(\tau_{1}\right)\right| \leq C_{2}^{(+)} e^{\kappa_{3} \tau_{1}}, \kappa_{3}>0, \tau_{1}<0, \\
\left|L_{0} u\left(\tau_{0}\right)\right| \leq C_{3}^{(-)} e^{-\kappa_{2} \tau_{0}}, \kappa_{2}>0, \tau_{0}>0, \\
\left|R_{0} u\left(\tau_{1}\right)\right| \leq C_{3}^{(+)} e^{\kappa_{3} \tau_{1}}, \kappa_{3}>0, \tau_{1}<0 .
\end{gathered}
$$

$\left[A_{5}\right]$ Suppose that the boundary data

$$
\begin{gathered}
\beta\left(t_{0}\right)-\varphi_{1,2}\left(t_{0}\right), \int_{0}^{t_{0}} h_{1}\left(\varphi_{1}(s)\right) \mathrm{d} s+\int_{t_{0}}^{T} h_{1}\left(\varphi_{2}(s)\right) \mathrm{d} s-\varphi_{1}(0), \\
\int_{0}^{t_{0}} h_{2}\left(\varphi_{1}(s)\right) \mathrm{d} s+\int_{t_{0}}^{T} h_{2}\left(\varphi_{2}(s)\right) \mathrm{d} s-\varphi_{2}(T),
\end{gathered}
$$

in the problems $Q_{0}^{(\mp)} J, L_{0} J$ and $R_{0} J$ belong to certain neighborhoods of the origin that guarantee the existence of solutions in these problems.

Remark 4.9 Assumption $\left[A_{5}\right]$ is analogous to Tikhonov's conditions, which require that the boundary data belong to the domains of influence of the corresponding asymptotically stable equilibria of the associated systems.

Remark 4.10 In the general case, the asymptotic approximation to the control is not an admissible control, since it drives the trajectory from the initial point only to an $O(\mu)$ neighborhood of the terminal point. For $t \in\left[0, t_{0}\right]$, The zero-order asymptotic solution is $Y_{0}=\varphi_{1}(t)+L_{0} y\left(\tau_{0}\right)+Q_{0}^{(-)} y(\tau), Y_{0}$ is not an admissible solution, as was shown in [12], we obtain

$$
Y_{0}(0, \mu)-y_{0 \mu}(0, \mu)=p_{0}(\mu) \neq 0, \quad Y_{0}\left(t_{0}, \mu\right)-y_{0 \mu}\left(t_{0}, \mu\right)=p_{1}(\mu) \neq 0,
$$

where $p_{i}(\mu)=O\left(e^{-\frac{t_{0}}{\mu}}\right), i=0,1$. To get an admissible solution $y_{0 \mu}$, we need to add a smoothing function $\theta_{0}(t, \mu)$, then $y_{0 \mu}=Y_{0}(t, \mu)+\theta_{0}(t, \mu), \quad u_{0 \mu}=\mu \frac{d y_{0 \mu}}{d t}$, where $\theta_{0}(t, \mu)=$ $A e^{-t / \mu}+B e^{\left(t-t_{0}\right) / \mu}$, while

$$
\begin{aligned}
& A=\left(-p_{0}(\mu)+e^{-t_{0} / \mu} p_{1}(\mu)\right)\left(1-e^{-2 t_{0} / \mu}\right)^{-1}, \\
& B=\left(-p_{1}(\mu)+e^{-t_{0} / \mu} p_{0}(\mu)\right)\left(1-e^{-2 t_{0} / \mu}\right)^{-1},
\end{aligned}
$$

EJQTDE, 2011 No. 46, p. 12 
$y_{0 \mu}$ is an admissible solution, since it satisfies the boundary condition, then we get the admissible control

$$
u_{0 \mu}=\mu \varphi_{1}^{\prime}(t)+L_{0} u\left(\tau_{0}\right)+Q_{0}^{(-)} u(\tau)-A e^{-t / \mu}+B e^{\left(t-t_{0}\right) / \mu}, t \in\left[0, t_{0}\right],
$$

similarly, we also have

$$
u_{0 \mu}=\mu \varphi_{2}^{\prime}(t)+Q_{0}^{(+)} u(\tau)+R_{0} u\left(\tau_{1}\right)-\bar{A} e^{-\left(t-t_{0}\right) / \mu}+\bar{B} e^{(t-T) / \mu}, t \in\left[t_{0}, T\right],
$$

where

$$
\begin{aligned}
& \bar{A}=\left(-\bar{p}_{0}(\mu)+e^{\left(t_{0}-T\right) / \mu} \bar{p}_{1}(\mu)\right)\left(1-e^{2\left(t_{0}-T\right) / \mu}\right)^{-1}, \\
& \bar{B}=\left(-\bar{p}_{1}(\mu)+e^{\left(t_{0}-T\right) / \mu} \bar{p}_{0}(\mu)\right)\left(1-e^{2\left(t_{0}-T\right) / \mu}\right)^{-1},
\end{aligned}
$$

It should be noted that the smoothing functions are exponentially small.

Then, we have so far constructed the leading terms

$$
\left\{\bar{y}_{0}^{*}(t), \quad \bar{u}_{0}^{*}(t)\right\}, \quad\left\{L_{0} y^{*}\left(\tau_{0}\right), \quad L_{0} u^{*}\left(\tau_{0}\right)\right\}, \quad\left\{Q_{0} y^{*}(\tau), \quad Q_{0} u^{*}(\tau)\right\}, \quad\left\{R_{0} y^{*}\left(\tau_{1}\right), \quad R_{0} u^{*}\left(\tau_{1}\right)\right\} .
$$

of asymptotic series for the problem (4.1) and (4.2). Additionally, we can obtain the minimum values of the corresponding optimal control problems $J_{0}^{*}, L_{0} J^{*}, Q_{0}^{(\mp)} J^{*}, R_{0} J^{*}$ :

$$
\begin{gathered}
J_{0}^{*}\left(\bar{u}_{0}\right)=\int_{0}^{T} f\left(\bar{y}_{0}^{*}, \bar{u}_{0}^{*}, t\right) d t \\
L_{0} J^{*}=\int_{y^{0}}^{\varphi_{1}(0)} \frac{\Delta_{0}^{(\mp)} f\left(\check{y}^{*}, \check{u}^{*}, 0\right)}{\check{u}^{*}} d \check{y}^{*}, \\
Q_{0}^{(\mp)} J^{*}= \pm \int_{\varphi_{1,2}\left(t_{0}\right)}^{\beta\left(t_{0}\right)} \frac{\Delta_{0}^{(\mp)} f\left(\tilde{y}^{(\mp) *}, \tilde{u}^{(\mp) *}, t_{0}\right)}{\tilde{u}^{(\mp) *}} d \tilde{y}^{*}, \\
R_{0} J^{*}=\int_{\varphi_{2}(T)}^{y^{T}} \frac{\Delta_{0}^{(\mp)} f\left(\hat{y}^{*}, \hat{u}^{*}, T\right)}{\hat{u}^{*}} d \hat{y}^{*},
\end{gathered}
$$

where

$$
\begin{array}{cc}
\check{y}^{*}=\varphi_{1}(0)+L_{0} y^{*}\left(\tau_{0}\right), & \check{u}=L_{0} u^{*}\left(\tau_{0}\right), \\
\hat{y}^{*}=\varphi_{2}(T)+R_{0} y^{*}\left(\tau_{1}\right), & \hat{u}^{*}=R_{0} u^{*}\left(\tau_{1}\right) .
\end{array}
$$

Next, we will show the validity of the formal solution.

Theorem 4.11 Suppose that $\left[A_{1}\right]-\left[A_{5}\right]$ hold. Then for sufficiently small $\mu>0$ there exists a step-like contrast structure solution $y(t, \mu)$ of the problem (2.1), moreover, the following asymptotic expansion holds

$$
y(t, \mu)=\left\{\begin{array}{l}
\varphi_{1}(t)+L_{0} y\left(\tau_{0}\right)+Q_{0}^{(-)} y(\tau)+O(\mu), 0 \leq t<t_{0}+\mu t_{1} \\
\varphi_{2}(t)+R_{0} y\left(\tau_{1}\right)+Q_{0}^{(+)} y(\tau)+O(\mu), t_{0}+\mu t_{1}<t \leq T
\end{array}\right.
$$


Proof of Theorem 4.11. In [12] the authors consider the existence of contrast structure for the following singularly perturbed differential equations with integral boundary conditions

$$
\left\{\begin{array}{l}
\mu \frac{d y}{d t}=z \\
\mu \frac{d z}{d t}=f(t, y) \\
y(0, \mu)=\int_{0}^{1} h_{1}(y(s, \mu)) d s, \quad y(1, \mu)=\int_{0}^{1} h_{2}(y(s, \mu)) d s .
\end{array}\right.
$$

It should be noted that the proof of Theorem 4.11 has no essential difference from that of Theorem 3.3 in [12], but some slight modifications, such as change $t \in[0,1]$ into $t \in[0, T]$, so we omit the details of the proof.

\section{Example}

In this section, an example is shown how to construct a zero order asymptotic solution with a step-like contrast structure. Consider the problem

$$
\left\{\begin{array}{l}
J[u]=\int_{0}^{2 \pi}\left(\frac{1}{4} y^{4}-\frac{1}{3} y^{3} \sin t-\frac{1}{2} y^{2}+y \sin t+\frac{1}{2} u^{2}\right) \mathrm{d} t \rightarrow \min _{u} \\
\mu \frac{\mathrm{d} y}{\mathrm{~d} t}=u \\
y(0, \mu)=\int_{0}^{2 \pi} y^{3}(s, \mu) d s, \quad y(2 \pi, \mu)=\int_{0}^{2 \pi} y^{5}(s, \mu) d s .
\end{array}\right.
$$

Let

$$
f(y, u, t)=\frac{1}{4} y^{4}-\frac{1}{3} y^{3} \sin t-\frac{1}{2} y^{2}+y \sin t+\frac{1}{2} u^{2},
$$

for every $t$, we have

$$
\begin{gathered}
\bar{y}_{0}(t)=\left\{\begin{array}{l}
-1, \quad 0 \leq t<\pi, \\
1, \quad \pi<t \leq 2 \pi
\end{array}\right. \\
\min _{\bar{y}} F\left(\bar{y}_{0}, t\right)= \begin{cases}-\frac{1}{4}-\frac{2}{3} \sin t, & 0 \leq t \leq \pi, \\
-\frac{1}{4}+\frac{2}{3} \sin t, & \pi \leq t \leq 2 \pi .\end{cases}
\end{gathered}
$$

The transition point $t_{0}=\pi$ is determined by the equation $\sin t_{0}=0$.

Firstly, we determine $Q_{0}^{(\mp)} y$ and $Q_{0}^{(\mp)} u$. By using of (3.6) and (3.7), $\bar{t}=\pi$, we get

$$
u^{2}-\left(\frac{1}{4} y^{4}-\frac{1}{2} y^{2}+\frac{1}{2} u^{2}\right)=\frac{1}{4}
$$

EJQTDE, 2011 No. 46, p. 14 
from our construction, we obtain the different orbit $S_{M_{1}}$ and $S_{M_{2}}$, which pass through the saddle points $M_{1}(\bar{t})$ and $M_{2}(\bar{t})$ respectively, have the form

$$
S_{M_{1}}: u^{(-)}=\frac{\sqrt{2}}{2}\left(1-y^{(-) 2}\right), \quad S_{M_{2}}: u^{(+)}=\frac{\sqrt{2}}{2}\left(1-y^{(+) 2}\right),
$$

by means of $\frac{d}{d \tau} Q_{0}^{(\mp)} y=Q_{0}^{(\mp)} u, \bar{u}_{0}(t)=0$, then the left and right zero-order terms of transition layer are determined by the following problems

$$
\frac{d Q_{0}^{(\mp)} y}{d \tau}=\frac{\sqrt{2}}{2}\left(1-\left(\mp 1+Q_{0}^{(\mp)} y\right)^{2}\right), \quad Q_{0}^{(\mp)} y(0)= \pm 1, \quad Q_{0}^{(\mp)} y(\mp \infty)=0 .
$$

Its solution are

$$
\begin{aligned}
& Q_{0}^{(-)} y=\frac{2 e^{\sqrt{2} \tau}}{1+e^{\sqrt{2} \tau}}, \quad Q_{0}^{(-)} u=\frac{2 \sqrt{2} e^{\sqrt{2} \tau}}{\left(1+e^{\sqrt{2} \tau}\right)^{2}} \\
& Q_{0}^{(+)} y=\frac{-2}{1+e^{\sqrt{2} \tau}}, \quad Q_{0}^{(+)} u=\frac{2 \sqrt{2} e^{\sqrt{2} \tau}}{\left(1+e^{\sqrt{2} \tau}\right)^{2}}
\end{aligned}
$$

Similarly, the problems to determine left and right boundary layer are

$$
\begin{gathered}
\frac{d L_{0} y}{d \tau_{0}}=-\frac{\sqrt{2}}{2}\left(1-\left(-1+L_{0} y\right)^{2}\right), \quad L_{0} y(0)=1, \quad L_{0} y(+\infty)=0, \frac{d L_{0} y}{d \tau_{0}}=L_{0} u . \\
\frac{d R_{0} y}{d \tau_{1}}=-\frac{\sqrt{2}}{2}\left(1-\left(1+R_{0} y\right)^{2}\right), \quad R_{0} y(0)=-1, \quad R_{0} y(-\infty)=0, \frac{d R_{0} y}{d \tau_{1}}=R_{0} u .
\end{gathered}
$$

so we have

$$
\begin{gathered}
L_{0} y=\frac{2 e^{-\sqrt{2} \tau_{0}}}{1+e^{-\sqrt{2} \tau_{0}}}, \quad L_{0} u=\frac{-2 \sqrt{2} e^{-\sqrt{2} \tau_{0}}}{\left(1+e^{-\sqrt{2} \tau_{0}}\right)^{2}}, \\
R_{0} y=\frac{-2 e^{\sqrt{2} \tau_{1}}}{1+e^{\sqrt{2} \tau_{1}}}, \quad R_{0} u=\frac{-2 \sqrt{2} e^{\sqrt{2} \tau_{1}}}{\left(1+e^{\sqrt{2} \tau_{1}}\right)^{2}} .
\end{gathered}
$$

Finally, the formal asymptotic solution is

$$
y(t, \mu)=\left\{\begin{array}{l}
-1+\frac{2 e^{-\sqrt{2} \tau_{0}}}{1+e^{-\sqrt{2} \tau_{0}}}+\frac{2 e^{\sqrt{2} \tau}}{1+e^{\sqrt{2} \tau}}+O(\mu), \quad 0 \leq t<\pi, \\
1+\frac{-2}{1+e^{\sqrt{2} \tau}}+\frac{-2 e^{\sqrt{2} \tau_{1}}}{1+e^{\sqrt{2} \tau_{1}}}+O(\mu), \quad \pi<t \leq 2 \pi .
\end{array}\right.
$$

\section{Acknowledgment}

The authors were grateful to the referees for their constructive comments which improve the presentation of the paper. The authors were supported by the Natural Science Foundation of China (No 11071075); National Laboratory of Biomacromolecules, Institute of Biophysics, Chinese Academy of Sciences; Natural Science Foundation of Shanghai(No 10ZR1409200), and in part by E-Institutes of Shanghai Municipal Education Commission(No N.E03004). 


\section{References}

[1] V. F. Butuzov and A. B. Vasil'eva, Asymptotic behavior of a solution of contrasting structure type, Mathematical Notes, vol. 42, no. 6, pp. 956-961, 1987.

[2] V. F. Butuzov, A. B. Vasil'eva and N. N. Nefedov, Asymptotic theory of contrast structures(Overview), Avtom. Telemekh, no. 7, pp. 4-32, 1997.

[3] A. B. Vasil'eva, V. F.Butuzov and N. N. Nefedov, Contrast structures in singularly perturbed problems, Fundam. Prikl. Mat, vol. 4, no. 3, pp.7 99-851, 1998.

[4] X. Lin, Heteroclinic bifurcation and singularly perturbed boundary value problems, Journal of Differential Equations, vol. 84, no. 2, pp. 319-382, 1990.

[5] X. Lin, Construction and asymptotic stability of structurally stable internal layer solutions, Transactions of the American Mathematical Society, vol. 353, no. 8, pp. 2983-3043, 2001.

[6] M. Ni and M. G. Dmitriev, Contrast structures in the simplest vector variational problem and their asymptotics, Avtom. Telemekh, no. 5, pp.41-52, 1998.

[7] A. B. Vasil'eva, M. G. Dmitriev and M. Ni, On a steplike contrast structure in a problem of variational calculus, Comput. Math. Math. Phys, vol. 44, pp. 1203-1212, 2004 .

[8] A. B. Vasil'eva, On contrast steplike structure for a system of singularly perturbed equations, Zh. Vychisl. Mat.Mat.Fiz, vol. 34, pp.1401-1411,1994.

[9] J. R. Cannon, The solution of the heat equation subject to the specification of energy, Quart. Appl. Math, vol. 21, no. 2, pp. 155-160, 1963.

[10] N. I. Ionkin, Solution of a boundary value problem in heat conduction theory with nonlocal boundary conditions, Differential Equations, vol. 13, pp. 294-304, 1977.

[11] F. Nicoud, T. Schonfeld, Integral boundary conditions for unsteady biomedical CFD applications, Internat. J. Numer. Methods Fluids, vol. 40, pp. 457-465, 2002.

[12] F. Xie, Z. Jin and M. Ni, On the step-type contrast structure of a second-order semilinear differential equation with integral boundary conditions, Electronic Journal of Qualitative Theory of Differential Equations, vol. 2010, no. 62, pp. 1-14,2010.

[13] S.V. Belokopytov and M. G.Dmitriev, Solution of classical optimal control problems with boundary layer, Avtom. Telemekh, no. 7, pp.71-82, 1989.

(Received April 8, 2011) 